\title{
Distribution of Hepatitis C Virus genotypes within in-and outpatients tested by the Immuno-Haematology and Blood Transfusion Service of the "Azienda Ospedaliera S. Maria, Terni" in the year 2012
}

\author{
Monica Proietti, Alessandra Pagnani, Francesco Trosino, Maria Chiara Medori, \\ Augusto Scaccetti \\ Servizio di Immunoematologia e Trasfusionale, Azienda Ospedaliera Santa Maria,Terni
}

Key Words: Hepatitis C Infection, HCV genotypes, RNA sequencing

Distribuzione dei genotipi dell'HCV nei pazienti afferenti al Servizio di Immunoematologia e Trasfusionale dell' Azienda Ospedaliera S. Maria di Terni nell'anno 20 I 2

\section{SUMMARY}

Background. The diffusion of Hepatitis CVirus genotypes in a specific geographical area plays a crucial role in public health defense management.

Objectives. In this study we evaluated the prevalence and distribution of the HCV genotypes in $204 \mathrm{HCV}-\mathrm{RNA}$ positive samples of outcoming and incoming patients of the Molecular Biology Laboratory of the ImmunoHaematology and Blood Transfusion Unit, AO “S. Maria", Terni, from Jenaury to December 2012.

Study Design. We have analized HCV genotypes prevalence and distribution in relation to qualitative variables such as sex, age, in- or out-coming.

Results. The results show that during the year 2012 the most prevalence genotype was genotype I (53.9\%). As far as the HCV subgenotypes, la and $3 a$ were prevalent within males $(25 \%$ and $20.1 \%$, respectively) and subgenotype Ib was most prevalent within females (9.3\%). In addition we observed also an increase in genotype $4(10.3 \%)$, probably due to the continuous migration of population from Northen-Africa and Middle-Eastern countries where such variant 4 is endemic. In regards to age, our data show that patients between the age of 30 and 50 years old, presented mainly HCV genotypes $3 \mathrm{a}(27 \%)$ and $4 \mathrm{c}(12.7 \%)$ whereas over 50 years old patients harboured more frequently genotypes Ib $(25.3 \%)$ and $2 \mathrm{a}(23.9 \%)$.

Conclusions. The high frequency of isolation of genotypes la and $3 \mathrm{a}$ agrees with previous observation made in patients from Northen and Central Italy where the infection with this viral variant is characteristic of young patients and is often associated with drug abuse. On the contrary, genotypes Ib and 2a seems to be more associated to community- acquired infections predominantly in blood transfused patients.

\section{INTRODUZIONE}

L'infezione da Virus dell'Epatite C (HCV) resta la principale causa di malattie croniche del fegato e lo studio della diffusione dei genotipi virali in una determinata area geografica svolge un ruolo cruciale nella difesa della salute pubblica (5).

Il genoma dell'HCV è costituito da una singola catena di RNA lineare a polarità positiva di circa 9400 nucleotidi che replica in stretta associazione con le membrane intracellulari (13) delle cellule infettate e funge direttamente da RNA-messaggero per la traduzione delle proteine virali.

L'RNA dell'HCV presenta un'elevata eterogeneità molecolare $\mathrm{e}$ una variabilità tale da indurre $\mathrm{i}$ ricercatori ad usare il termine di "quasispecie" virale $(6,11)$.
Il maggior grado di variabilità si osserva nelle regioni che codificano per proteine la cui struttura non è sottoposta a stretti vincoli funzionali, come nella zona che codifica per l'envelope virale. Altre regioni, come quella del Core e dell'estremità 5', 5'Un-Traslated Region (5'UTR), sono più conservate. In base al grado di analogia delle sequenze l'HCV viene differenziato in genotipi, sottotipi, isolati e "quasispecie" $(7,9,17,18)$.

La classificazione attualmente accettata è quella proposta da Simmonds et al, che prevede la comparazione di sequenze derivate da diverse regioni genomiche.

La distribuzione geografica dei genotipi è variabile: la prevalenza dell'1b è alta in Europa e Giappone, ma più bassa in America dove predo-

\section{Corresponding author: Proietti Monica}

Servizio di Immunoematologia e Trasfusionale, Azienda Ospedaliera Santa Maria,Terni

Via Tristano di Joannuccio I, 05100 Terni, Italy - Tel.0744205529 - Fax 0744205846

E-mail: monicaproietti@aruba.it 
mina l'1a. Il genotipo 4 è stato trovato soprattutto in Africa e Medio Oriente, il 5 in Sud Africa ed il 6 in Estremo Oriente $(4,17,19)$.

Essendo l'HCV un virus a trasmissione parenterale, studi sono stati intrapresi per evidenziare associazione tra genotipo e via di trasmissione.

L'1b ed il $2 \mathrm{a} / 2 \mathrm{c}$ sembrano prevalere tra i soggetti trasfusi o con infezione "sporadica", mentre i genotipi 1a e 3 a sono $i$ più diffusi tra $i$ tossicodipendenti per via endovenosa $(10,12)$.

E stata notata una variazione dei genotipi con l'età anagrafica, essendo il genotipo $1 \mathrm{~b}$ e $2 \mathrm{a} / 2 \mathrm{c}$ presente soprattutto nei soggetti più anziani rispetto all'1a e 3 a, prevalenti nei giovani $(12,16)$.

Infine, alcuni studi hanno osservato una variazione della prevalenza nel tempo dei genotipi $1 \mathrm{~b}$ e $2 \mathrm{a} / 2 \mathrm{c}$ nei soggetti trasfusi, con una diminuzione dell' $1 \mathrm{~b}$ ed un aumento del $2 \mathrm{a} / 2 \mathrm{c}$ (12).

Tali dati non sono stati confermati da altri Autori (15). Concordanza sembra invece esserci nel considerare il 3 a come il genotipo a più recente diffusione, rispetto all' $1 \mathrm{~b}$ e $2 \mathrm{a} / 2 \mathrm{c}(12,15)$.

\section{MATERIALI E METODI}

Nell'anno 2012 sono pervenute presso il Laboratorio di Biologia Molecolare del Servizio di Immunoematologia e Trasfusionale (SIT) dell'Azienda Ospedaliera "S. Maria" di Terni, 341 richieste ospedaliere ed ambulatoriali di genotipizzazione del virus dell'HCV.

Ciascun campione di sangue, raccolto in apposite provette sterili con anticoagulante acido Etilendiamminotetraacetico (EDTA), è stato centrifugato entro 6 ore dal prelievo, per 20 minuti a 3000 RPM (800-1600 x g) a temperatura ambiente. Il plasma così ottenuto è stato suddiviso in tre aliquote da $1 / 1.5 \mathrm{~mL}$ e conservato in provette di polipropilene sterili (SARSTED) a $-80^{\circ} \mathrm{C}$ fino al momento dell'esecuzione dei test.

Una ulteriore aliquota di plasma è stata analizzata per la ricerca degli anticorpi anti-HCV con un metodo immunoenzimatico su piastra (Test MUREX HCV Serotyping, ABBOTT Murex) o con dosaggio immunologico chemiluminescente a cattura CHEMIFLEX (chemiluminescenza perfezionata a protocolli flessibili) o CMIA (Chemiluminescent Microparticle ImmunoAssay) Architect System Anti-HCV (Architect System ABBOTT Diagnostic Division, Wiesbaden, Germany).

La viremia è stata valutata in Real Time con i reattivi per la quantificazione dell'acido nucleico virale COBAS AmpliPrep/COBAS TaqMan HCV Test, v. 2.0 Roche Diagnostics.

Il test utilizzato nel Laboratorio di Biologia Molecolare del SIT di Terni permette la quantificazione di HCV-RNA con intervallo lineare del- l'analisi da $15 \mathrm{UI} / \mathrm{mL}$ a $1.00 \mathrm{E}+08 \mathrm{UI} / \mathrm{mL}$.

La genotipizzazione dell'HCV è stata effettuata mediante sequenziamento (metodo di Sanger).

Il test utilizzato in questo lavoro è stato il TRUGENE HCV 5'NC Genotyping Kit (Siemens Healthcare Diagnostics Inc. Tarrytown, NY USA) che impiega amplificati PCR della regione 5'UTR compresi tra i nucleotidi 254 e 378 . Tale kit impiegato insieme al sistema Open Gene DNA Sequencing System, permette di ottenere sequenze bidirezionali nella regione 5'UTR del virus dell'epatite C (8). Il protocollo di genotipizzazione prevede l'utilizzo dell'amplicone precedentemente generato dal test Roche Cobas Amplicor ${ }^{\mathrm{TM}}$ $\mathrm{HCV}$, che corrisponde ad un frammento di 244 nucleotidi interno alla regione 5' UTR del virus.

Il sequenziamento diretto degli ampliconi Roche prevede come primo passaggio la neutralizzazione dell'amplificato seguita dalla purificazione dello stesso per mezzo del QIAquick PCR Purification Kit (Qiagen SA, Countaboeuf, France).

I prodotti purificati rappresentano il materiale di partenza per la "CLIP ${ }^{\mathrm{TM}}$ Sequencing". Questo sequenziamento chimico produce simultaneamente ampliconi target bi-direzionali mediante cicli di PCR, attraverso l'uso di due primers oligonucleotidici marcati con differenti fluorocromi (Cy5 e Cy5.5). Il sequenziamento automatizzato del DNA del frammento di 183-bp prodotto dalla reazione CLIP ${ }^{\mathrm{TM}}$, è ottenuto dopo 25 minuti di corsa elettroforetica sulla Long-Read Tower del Sequenziatore Siemens.

Le sequenze forward e reverse di ciascuna coppia, vengono infine combinate ed automaticamente allineate e comparate con le sequenze di riferimento immagazzinate nell'HCV "GeneLibrarian" software Module, in modo da determinare il tipo, sottotipo ed isolato del virus infettante (14).

Il sistema informatico TRUGENE HCV 5'NC Module (3.1.2) è un database che contiene centinaia di sequenze caratterizzate precedentemente e che viene periodicamente aggiornato. La libreria di riferimento per i genotipi valutati in questo studio è stata la libreria 5'UTR 0902.

Le analisi statistiche dei dati sono state effettuate utilizzando il programma GraphPad Prism, versione 5.0.

L'associazione tra le variabili qualitative ed i genotipi virali è stata stimata con il test del $\chi^{2}$ (o $\chi^{2}$ Fisher's exact Test) a due code con intervallo di confidenza del 95\%.

Dopo aver creato una tabella di contingenza, la significatività dell'associazione tra le variabili ed i genotipi è stata stimata riportando l'indice di significatività $p$ (associazione statisticamente significativa per valori di $p$ inferiori a 0.05 ). 


\section{RISULTATI}

Delle 341 richieste ospedaliere ed ambulatoriali di genotipizzazione del virus dell'HCV pervenute al Laboratorio di Biologia Molecolare, solo quelle relative a 204 pazienti presentavano valori di viremia tali da poter consentire l'esecuzione del test di tipizzazione del genoma mediante sequenziamento. Le restanti 137 erano relative a pazienti con bassa viremia o addirittura con HCV-RNA non rilevato, condizioni, entrambe, che non hanno consentito l'esecuzione del sequenziamento.

Dei 204 campioni sequenziati, 149 (73\%) appartenevano a pazienti di sesso maschile e 55 (23\%) a pazienti di sesso femminile come illustrato in Tabella 1. La provenienza di tali campioni è descritta in Tabella 1 e mostra una netta prevalenza dei pazienti provenienti dal Sert di Terni (50 campioni), seguiti dai campioni provenienti dal Day Hospital delle Malattie Infettive (43 campioni), dai pazienti comunitari extra-ospedalieri o Esterni (17 campioni), dalla Casa Circondariale di Terni (15 campioni) e dai centri raccolta dell'Azienda Sanitaria Locale $n^{\circ} 4$ (ASL $\left.n^{\circ} 4\right)$ dislocati in tutto il territorio della Provincia di Terni (15 campioni). La distribuzione dei genotipi e dei sottotipi dei campioni di plasma analizzati è riportata nella Tabella 2.

Dall'analisi effettuate, il genotipo 1 è risultato il più rappresentato $(53.9 \%)$ seguito dal genotipo 3 $(24 \%)$, dal genotipo $2(11.3 \%)$ e dal genotipo 4 (10.3\%). Nell'anno 2012 i genotipi 5 e 6 non sono mai stati rilevati; d'altra parte, la diffusione del genotipo 5, limitata al Sud Africa e quella del genotipo 6 al Sud-Est Asiatico (1), ne rende difficile il riscontro nel territorio Italiano.

Nella Tabella 3 viene descritta la distribuzione dei genotipi e dei sottotipi virali dell'HCV relativi alla Provincia di Terni nell'anno 2012, tenendo in considerazione il sesso dei pazienti.

Dall'analisi della Tabella 3, le frequenze statisticamente rilevanti sono state quelle relative all'associazione tra uomini-donne/genotipo 1a (indice di significatività $p=0.026$ ), tra uomini-donne/genotipo $1 \mathrm{~b}(p=0.014)$, tra uomini-donne/genotipo $2 \mathrm{a}$ $(p=0.0006)$ in quanto tutte le altre associazioni hanno presentato valori di $p \geq 0.05$. Nei dati del 2012 relativi alla realtà della Provincia di Terni, ed illustrati nella Tabella 2 il sottotipo 1a è risultato il più frequente essendo stato riscontrato in 61 (29.9\%) pazienti a conferma della sua notevole prevalenza nell'Italia Centro-Settentrionale (2). Rilevante è stata la frequenza del genotipo 3a (49 campioni pari al 24\%) e del genotipo $1 \mathrm{~b}$ (41 campioni pari al 20.1\%) in accordo con altre indagini relative all'Italia centrale, che vedono il genotipo 1 essere prevalente in queste zone (3). Dalla Tabella 2 si evince che la distribuzione degli altri sottotipi virali rilevati nell'anno 2012 risulta essere, in ordine decrescente: sottotipo 2a (19 campioni pari al 9.3\%), sottotipo 4c (17 campioni pari all' $8.3 \%$ ), sottotipo $1 \mathrm{a} / 1 \mathrm{~b}$ (8 campioni pari al $3.9 \%$ ), sottotipo 2 b (3 campioni pari all'1.5\%), sottotipi $2 \mathrm{a} / 2 \mathrm{~d}, 4 \mathrm{a} / 4 \mathrm{c} / 4 \mathrm{e} / 41,4 \mathrm{c} / 4 \mathrm{e} / 41,4 \mathrm{c} / 4 \mathrm{t}, 4 \mathrm{e},(1$ campione per sub-tipo con percentuale pari allo $0.5 \%$ ciascuno). Come evidenziato nella Tabella 2, per un campione (HCV-RNA genotipo $1 \mathrm{~g}+4 \mathrm{a} / 4 \mathrm{c} / 4 \mathrm{r}$ ) non è stato possibile determinare l'esatto genotipo virale a causa della bassa similarità tra gli isolati riscontrati nel plasma e le sequenze genomiche presenti nella libreria 5'UTR 0902; occorre dunque precisare che non è sempre possibile determinare il sottotipo dell'HCV-RNA sequenziando la regione 5'UTR del genoma virale. In questi casi per determinare il sottotipo dell'HCV-RNA occorrerebbe utilizzare un'altra regione genomica da sequenziare quale, ad esempio, la regione NS5B.

Dalla fine di ottobre 2006 il Laboratorio di Biologia Molecolare esegue analisi non solo per i pazienti ricoverati e per quelli afferenti al poliambulatorio esterno dell'Azienda Ospedaliera "S. Maria", ma anche per i pazienti in precedenza utenti della Asl $n^{\circ} 4$ di Terni e quindi analizza campioni provenienti dai Sert, dalle Comunità di Recupero e dalle Case Circondariali territoriali. Tale situazione potrebbe aver determinato il cambiamento nella prevalenza dei genotipi, a causa dell'abbassamento dell'età anagrafica dei pazienti considerati. Per cercare un riscontro significativo a questa ipotesi, i 204 campioni esaminati per la tipizzazione del genoma dell'HCV nell'anno 2012 sono stati successivamente suddivisi in tre classi di età: > di 50 anni, tra 50 e 30 anni, uguale o inferiore a 30 anni (Tabella 4).

Dei dati riportati in Tabella 4 le associazioni classi d'età/genotipo relative al sottotipola $(p=0.011)$, $1 \mathrm{a} / 1 \mathrm{~b}(p=0.033), 2 \mathrm{a}(p<0.0001)$ e $4 \mathrm{c}(p=0.0072)$, hanno corrisposto al criterio di significatività, le altre associazioni sono risultate statisticamente non significative $(p \geq 0.05)$; alla significatività statistica dei dati relativi ai genotipi $1 \mathrm{a}, 1 \mathrm{a} / 1 \mathrm{~b}, 2 \mathrm{a}$ e $4 \mathrm{c}$ hanno contribuito principalmente le due classi di età $>$ di anni 50 e tra 50 e 30 anni, essendo il contributo numerico dei pazienti con età $\leq$ di 30 anni (11 su 204 totali) di gran lunga inferiore. Si è quindi proceduto alla valutazione del rapporto tra le variabili qualitative (classi d'età: $>50$ anni e 5030 anni) e genotipi 1a, 1a/1b, 2a, 4c come descritto in Tabella 5.

Tutti i valori di $p$ sono risultati minori di $0.05 \mathrm{e}$ quindi tutte le correlazioni tra genotipi virali ed età riportate in Tabella 5 sono risultate statisticamente significative. Il valore di $p=0.028$ per il sottotipo 1a sta ad indicare che la maggior parte dei 
pazienti tra 30-50 anni presenta questo genotipo. Il valore di $p=0.0047$ per il sottotipo $4 \mathrm{c}$ evidenzia che su 17 campioni totali che avevano questa variante virale, 16 appartenevano a pazienti con età compresa fra 30-50 anni. Ugualmente, il valore di $p<0.0001$ per il sottotipo $2 \mathrm{a}$, evidenzia come questo genotipo sia rappresentato nei pazienti più anziani ( $>50$ anni). Il valore di $p=0.0218$ per il sottotipo $1 \mathrm{a} / 1 \mathrm{~b}$, si spiega collocando nella fascia d'età $>50$ anni il $75 \%$ dei pazienti con genotipo $1 \mathrm{a} / 1 \mathrm{~b}$.

\section{DISCUSSIONE}

I dati relativi all'anno 2012 ottenuti dai 204 campioni analizzati evidenziano una distribuzione dei genotipi dell'HCV in buona misura sovrapponibile alla diffusione delle varianti del virus nella popolazione generale Italiana ed in particolare in quella dell'Italia Centrale.

Il genotipo più frequente è risultato essere il genotipo 1 in accordo con precedenti indagini condotte sia nel territorio nazionale che nelle regioni del Centro Italia $(2,3)$. Per quanto concerne la preva- lenza dei vari sottogenotipi dell'HCV, il sottotipo $1 \mathrm{a}$ è risultato il più rappresentato soprattutto fra $\mathrm{i}$ soggetti di sesso maschile; i sottotipi $3 \mathrm{a}$ ed $1 \mathrm{~b}$ (la cui associazione con le pazienti di sesso femminile è risultata statisticamente significativa) sono risultati parimenti molto frequenti.

I dati del 2012 mostrano inoltre un incremento del genotipo 4 probabilmente dovuto alla crescita dell'immigrazione dal Nord-Africa e dal MedioOriente aree geografiche ad elevata endemia per tale genotipo. Considerando, infine, la variabile qualitativa età anagrafica dei pazienti, i soggetti con più di 50 anni avevano soprattutto i genotipi $2 \mathrm{a}$ e $1 \mathrm{~b}$ mentre nei soggetti con età compresa tra $\mathrm{i}$ 50 ed i 30 anni i genotipi più frequenti sono risultati essere 1'1a, il 3a ed il 4c.

La presenza nella nostra realtà dei genotipi 1a e $3 a$ potrebbe far pensare, come in altre parti d'Italia, ad un legame con la diffusione e l'abuso di sostanze stupefacenti, mentre i genotipi $1 \mathrm{~b}$ e $2 \mathrm{a}$ sembrerebbero essere associati ad infezioni post-trasfusionali e soprattutto "community-acquired".

Tabella I. Campioni sequenziati nella regione 5'UTR del genoma virale nell'anno 2012 distinti per sesso e reparto di provenienza

\begin{tabular}{|c|c|c|c|c|}
\hline Reparto di provenienza & UOMINI & DONNE & Totale & Percentuale\% \\
\hline $\begin{array}{l}\text { ACOIN (Amelia "Comunità Incontro" di } \\
\text { recupero per tossicodipendenti) }\end{array}$ & 6 & $\mathrm{I}$ & 7 & 3.4 \\
\hline ASL $n^{\circ} 4$ & 8 & 7 & 15 & 7.3 \\
\hline Casa Circondariale Terni & 15 & & 15 & 7.3 \\
\hline Clinica Medica Generale & 1 & $\mathrm{I}$ & 2 & 1.0 \\
\hline Dermatologia & & $\mathrm{I}$ & 1 & 0.5 \\
\hline DH Malattie Infettive & 28 & 15 & 43 & 21.1 \\
\hline DH Medico & 2 & 2 & 4 & 2.0 \\
\hline DH Neurologico & $\mathrm{I}$ & & 1 & 0.5 \\
\hline Esterni & 11 & 6 & 17 & 8.3 \\
\hline Malattie Apparato Respiratorio (MAR) & & 1 & 1 & 0.5 \\
\hline Malattie Infettive & 4 & 3 & 7 & 3.4 \\
\hline Medicina d'Urgenza & $\mathrm{I}$ & & $\mathrm{I}$ & 0.5 \\
\hline Medicina Interna $A / B$ & 1 & & $\mathrm{I}$ & 0.5 \\
\hline Nefrologia & & 1 & $\mathrm{I}$ & 0.5 \\
\hline Oncoematologia & 2 & 1 & 3 & 1.5 \\
\hline Ospedale Amelia & 5 & 4 & 9 & 4.4 \\
\hline Ospedale Narni & 4 & $\mathrm{I}$ & 5 & 2.4 \\
\hline Ospedale Orvieto & 5 & 7 & 12 & 5.9 \\
\hline Sert Narni & 4 & & 4 & 2.0 \\
\hline Sert Orvieto & 2 & & 2 & 1.0 \\
\hline Sert Terni & 48 & 2 & 50 & 24.5 \\
\hline Servizio Trasfusion. Terni & & 1 & 1 & 0.5 \\
\hline Terap. Intens. Post Operat. & & $\mathrm{I}$ & 1 & 0.5 \\
\hline Unità del Fegato & 1 & & 1 & 0.5 \\
\hline TOTALE & 149 & 55 & 204 & 100.0 \\
\hline
\end{tabular}


Tabella 2. Campioni sequenziati nella regione 5'UTR del genoma dell'HCV nell'anno 20I2: distribuzione dei genotipi e dei sottotipi virali

\begin{tabular}{|c|c|c|c|}
\hline GENOTIPI & Sottotipi & $\mathbf{N}^{\circ}$ Campioni & Percentuale \% \\
\hline \multirow{3}{*}{ Genotipo I } & Ia & 61 & 29.9 \\
\hline & $\mathrm{Ib}$ & 41 & 20.1 \\
\hline & $\mathrm{la} / \mathrm{lb}$ & 8 & 3.9 \\
\hline \multirow{3}{*}{ Genotipo 2} & $2 \mathrm{a}$ & 19 & 9.3 \\
\hline & $2 a / 2 d$ & 1 & 0.5 \\
\hline & $2 \mathrm{~b}$ & 3 & 1.5 \\
\hline Genotipo 3 & $3 a$ & 49 & 24.0 \\
\hline \multirow{5}{*}{ Genotipo 4} & $4 \mathrm{a} / 4 \mathrm{c} / 4 \mathrm{e} / 4$ & 1 & 0.5 \\
\hline & $4 c$ & 17 & 8.3 \\
\hline & $4 \mathrm{c} / 4 \mathrm{e} / 4 \mathrm{l}$ & 1 & 0.5 \\
\hline & $4 \mathrm{c} / 4 \mathrm{t}$ & 1 & 0.5 \\
\hline & $4 \mathrm{e}$ & 1 & 0.5 \\
\hline Genotipo bassa similarità* & $\lg / 4 \mathrm{a} / 4 \mathrm{c} / 4 \mathrm{r}$ & 1 & 0.5 \\
\hline \multicolumn{2}{|c|}{$\begin{array}{ll}\text { TOTALE } \\
\end{array}$} & 204 & 100.0 \\
\hline
\end{tabular}

*Non è sempre possibile determinare il genotipo/sottotipo HCV utilizzando la regione 5'UTR. In questi casi per definire l'esatto genotipo/sottotipo HCV occorrerebbe utilizzare un'altra regione del genoma virale.

Tabella 3. Campioni sequenziati nella regione 5'UTR del genoma virale nell'anno 2012: distribuzione dei genotipi e dei sottotipi virali nei pazienti di sesso maschile e femminile

\begin{tabular}{|c|c|c|c|c|c|}
\hline GENOTIPI & Sottotipi & Uomini & Donne & $\%$ Uomini & \% Donne \\
\hline \multirow{3}{*}{ Genotipo I } & la & 51 & 10 & 25 & 4.9 \\
\hline & $\mathrm{Ib}$ & 22 & 19 & 10.8 & 9.3 \\
\hline & $\mathrm{Ia} / \mathrm{Ib}$ & 6 & 2 & 2.9 & 1.0 \\
\hline \multirow{3}{*}{ Genotipo 2} & $2 \mathrm{a}$ & 7 & 12 & 3.4 & 5.9 \\
\hline & $2 a / 2 d$ & & $\mathrm{I}$ & & 0.5 \\
\hline & $2 b$ & 3 & & 1.5 & \\
\hline Genotipo 3 & $3 a$ & 41 & 8 & 20.1 & 3.9 \\
\hline \multirow{5}{*}{ Genotipo 4} & $4 \mathrm{a} / 4 \mathrm{c} / 4 \mathrm{e} / 4 \mathrm{I}$ & $\mathrm{I}$ & & 0.5 & \\
\hline & $4 c$ & 14 & 3 & 6.8 & 1.5 \\
\hline & $4 c / 4 e / 41$ & $\mathrm{I}$ & & 0.5 & \\
\hline & $4 c / 4 t$ & 1 & & 0.5 & \\
\hline & $4 e$ & 1 & & 0.5 & \\
\hline $\begin{array}{l}\text { Genotipo bassa } \\
\text { similarità }\end{array}$ & $\mathrm{Ig} / 4 \mathrm{a} / 4 \mathrm{c} / 4 \mathrm{r}$ & 1 & & 0.5 & \\
\hline \multicolumn{2}{|c|}{ TOTALE } & 149 & 55 & 73 & 27 \\
\hline
\end{tabular}

Tabella 4. Distribuzione dei genotipi dell'HCV in relazione a tre grandi classi d'età: superiore a 50 anni, tra 50 e 30 anni ed inferiore o uguale a 30 anni

\begin{tabular}{|c|c|c|c|c|c|c|c|c|c|c|c|c|c|c|}
\hline $\begin{array}{l}\text { Sottotipi } \\
\text { virali }\end{array}$ & Ia & Ib & $\begin{array}{l}\mathrm{I} \mathbf{a} / \\
\mathrm{Ib}\end{array}$ & $2 a$ & $\begin{array}{l}2 \mathrm{a} / \\
2 \mathrm{~d}\end{array}$ & $2 b$ & $3 a$ & $\begin{array}{c}4 a / 4 c l \\
4 e / 4 I\end{array}$ & $4 c$ & $\begin{array}{c}4 \mathrm{cl} \\
4 \mathrm{e} / \\
4 \mathrm{l}\end{array}$ & $\begin{array}{l}4 \mathrm{cl} \\
4 \mathrm{t}\end{array}$ & $4 e$ & $\begin{array}{c}1 \mathrm{~g}+ \\
4 \mathrm{a} / \\
4 \mathrm{e} / \\
\mathbf{4 r}\end{array}$ & $\begin{array}{l}\text { Tot. } \\
\text { (\%) }\end{array}$ \\
\hline$>50$ anni & $\begin{array}{c}12 \\
(5.9)\end{array}$ & $\begin{array}{c}17 \\
(8.3)\end{array}$ & $\begin{array}{c}6 \\
(2.9)\end{array}$ & $\begin{array}{c}16 \\
(7.8)\end{array}$ & $\begin{array}{c}1 \\
(0.5)\end{array}$ & $\begin{array}{c}2 \\
(1.0)\end{array}$ & $\begin{array}{c}12 \\
(5.9)\end{array}$ & & $\begin{array}{c}1 \\
(0.5)\end{array}$ & & & & & $\begin{array}{c}67 \\
(32.8)\end{array}$ \\
\hline $50-30$ anni & $\begin{array}{c}43 \\
(21.1)\end{array}$ & $\begin{array}{c}22 \\
(10.8)\end{array}$ & $\begin{array}{c}2 \\
(1.0)\end{array}$ & $\begin{array}{c}3 \\
(1.5)\end{array}$ & & $\begin{array}{c}1 \\
(0.5)\end{array}$ & $\begin{array}{c}34 \\
(16.7)\end{array}$ & $\begin{array}{c} \\
(0.5)\end{array}$ & $\begin{array}{c}16 \\
(7.8)\end{array}$ & $\begin{array}{c}1 \\
(0.5)\end{array}$ & $\begin{array}{c}1 \\
(0.5)\end{array}$ & $\begin{array}{c}1 \\
(0.5)\end{array}$ & $\begin{array}{c}1 \\
(0.5)\end{array}$ & $\begin{array}{c}126 \\
(61.8)\end{array}$ \\
\hline$\leq 30$ anni & $\begin{array}{c}6 \\
(2.9) \\
\end{array}$ & $\begin{array}{c}2 \\
(1.0)\end{array}$ & & & & & $\begin{array}{c}3 \\
(1.5)\end{array}$ & & & & & & & $\begin{array}{c}11 \\
(5.4)\end{array}$ \\
\hline $\begin{array}{c}\text { Totale } \\
\text { (\%) }\end{array}$ & $\begin{array}{c}61 \\
(29.9)\end{array}$ & $\begin{array}{c}41 \\
(20.1)\end{array}$ & $\begin{array}{c}8 \\
(3.9)\end{array}$ & $\begin{array}{c}19 \\
(9.3)\end{array}$ & $\begin{array}{c}1 \\
(0.5)\end{array}$ & $\begin{array}{c}3 \\
(1.5)\end{array}$ & $\begin{array}{c}49 \\
(24.0)\end{array}$ & $\begin{array}{c}1 \\
(0.5)\end{array}$ & $\begin{array}{l}17 \\
(8.3)\end{array}$ & $\begin{array}{c}1 \\
(0.5)\end{array}$ & $\begin{array}{c}1 \\
(0.5)\end{array}$ & $\begin{array}{c}1 \\
(0.5)\end{array}$ & $\begin{array}{c}1 \\
(0.5)\end{array}$ & $\begin{array}{c}204 \\
(100)\end{array}$ \\
\hline
\end{tabular}

Tabella 5. Associazioni statisticamente significative genotipi-classi d'età

\begin{tabular}{|l|c|c|c|c|c|}
\hline genotipo & Ia & Ia/Ib & 2a & 4c & Totale (\%) \\
\hline$>50$ anni & $12(34.3)$ & $6(17.1)$ & $16(45.7)$ & I (2.9) & $35(100)$ \\
\hline $50-30$ anni & $43(67.2)$ & $2(3.1)$ & $3(4.7)$ & $16(25.0)$ & $64(100)$ \\
\hline $0,05 \geq \mathrm{p}<0,05$ & 0,0028 & 0,0218 & $<0,0001$ & 0,0047 & \\
\hline
\end{tabular}




\section{BIBLIOGRAFIA}

1. Argentini $\mathrm{C}$, Dettori $\mathrm{S}$, Rapicetta $\mathrm{M}$. Variabilità virale ed infezione da HCV. Rapporti ISTSAN 2003; 03/09: 116-20.

2. Bellentani S, Mignoli L, BedogniG, Croce LS, Tribelli C. Epidemiology of hepatitis $\mathrm{C}$ virus infection. Minerva Gastroenterol Dietol 2005; 51: 15-29.

3. Cenci M, De Soccio G, Recchia O. Prevalence of hepatitis $\mathrm{C}$ virus (HCV) genotypes in central Italy. Anticancer Res 2003; 23: 5129-32.

4. Cicciarello S, Borgia G, Crowell J, et al. Prevalence of hepatitis $\mathrm{C}$ virus genotypes in Southern Italy. Eur $J$ Epidemiol 1997; 13(1): 49-54.

5. Marascio N, Matera G, Quirino A, et al. Eleven-year distribution pattern of hepatitis $\mathrm{C}$ virus in southern Italy. J Pathog 2012; 2012: 631095.

6. Martell M, Esteban JI, Quer J, et al. Hepatitis C virus (HCV) circulates as a population of different but closely related genomes: quasispecies nature of $\mathrm{HCV}$ genome distribution. J Virol 1992; 66: 3225-9.

7. Miyakawa Y, Okamoto H, Mayumi M. Classifying hepatitis C virus genotypes. Mol Med Today 1995; 1: 20-5.

8. Nolte FS, Green AM, Fiebelkorn KR, et al. Clinical evaluation of two methods for genotyping hepatitis $\mathrm{C}$ virus based an analysis of the 5' non-coding region. $J$ Clin Microbiol Apr 2003; 41(4): 1558-64.

9. Okamoto H, Okada S, Sugiyama Y, et al. Nucleotide sequence of genomic RNA of hepatitis $\mathrm{C}$ virus isolated from a human carrier: comparison with reported isolates for conserved and divergent regions. $J$ Gen Virol 1991; 72: 2697-704.

10. Pawlotsky JM, Tsakiris L, Roudot-Thoraval F, et al. Relationship between hepatitis $\mathrm{C}$ virus genotypes and sources of infection in patients with chronic hepatitis C. J Infectious Dis 1995; 171: 1607-10.
11. Plauzolles A, Lucas M, Gaudieri S. Hepatitis C virus adaptation to T-cell immune pressure. Scientific World Journal 2013; 2013: 673240.

12. Roffi L, Ricci A, Ogliari C, et al. HCV genotypes in Northern Italy: a survey of 1368 histologically proven chronic hepatitis C patients. J Hepatol 1998; 29: 701-6.

13. Romero-Brey I, Merz A, Chiramel A, et al. Threedimensional architecture and biogenesis of membrane structures associated with hepatitis $\mathrm{C}$ virus replication. PLoS Pathog 2012 Dec; 8(12): e1003056.

14. Roque-Afonso AM, Férey MP, Poveda JD, Marchadier E, Dussaix E. Performance of TRUGENE $^{\text {TM }}$ hepatitis C virus 5' non coding genotyping kit, a new CLIP ${ }^{\mathrm{TM}}$ sequencing-based assay for hepatitis C virus genotype determination. $J$ of Viral Hepatitis 2002; 9: 385-9.

15. Sacco R, Randone A, Flichman D, et al. The prevalence of hepatitis $C$ virus types in patients of the same geographic area, according to the source of infection and liver disease. Clin Diagn Virol 1997; 8(3): 189-94.

16. Silini E, Bono F, Cividini A, et al. Molecular epidemiology of hepatitis $\mathrm{C}$ virus infection among intravenous drug users. J Hepatol 1995; 22: 691-5.

17. Simmonds P, Holmes EC, Cha TA, et al. Classification of hepatitis $\mathrm{C}$ virus into six major genotypes and a series of subtypes by phylogenetic analysis of the NS5 region. J Gen Virol 1993; 74: 2391-9.

18. Simmonds P, Smith DB, McOmish F, et al. Identification of genotypes of hepatitis $\mathrm{C}$ virus by sequence comparison in the core, E1 and NS-5 regions. J Gen Virol 1994; 75: 1053-61.

19. Xu LZ, Larzul D, Delaporte E, Bréchot C, Kremsdorf D. Hepatitis $\mathrm{C}$ virus genotype 4 is highly prevalent in central Africa (Gabon). J Gen Virol 1994; 75(Pt 9): 2393-8. 